\title{
PRIMEIRAS DISSERTAÇÕES DO CURSO DE MESTRADO DA ESCOLA DE ENFERMAGEM ANNA NERY (1972-1975)
}

First dissertations of the Anna Nery nursing school master's degree program (19721975)

Primeras disertaciones del curso de maestría de la escuela de enfermería Anna Nery (1972-1975)

Carolina Ribeiro Machado

leda de Alencar Barreira ${ }^{2}$

Ana Lia Trindade Martins ${ }^{3}$

\section{RESUMO}

Estudo histórico-social que tem como objeto as condições de produção das primeiras dissertações do Curso de Mestrado da Escola de Enfermagem Anna Nery, elaboradas no período 1972-1975. Objetivos: descrever as características iniciais do Curso de Mestrado da EEAN; analisar o perfil das autoras das primeiras dissertações desse Curso e de suas orientadoras; e discutir a inserção dessas dissertações nas áreas e linhas de pesquisa em enfermagem. Foi realizada análise documental e foram organizados quadros para apresentação dos resultados. Resultados: nove das doze alunas de tempo integral da turma pioneira apresentaram suas disser tações antes do prazo regulamentar de quatro anos. Embora oito das mestrandas fossem docentes, cerca de $90 \%$ de suas dissertações inserem-se na Área Assistencial, foco de interesse de sua prática de ensino. 0 mestrado da EEAN qualificou profissionais para lecionar nos cursos superiores de enfermagem. As nove primeiras dissertações nele produzidas constituíram importante contribuição para a produção científica de enfermagem.

Palavras-chave: História da Enfermagem. Pesquisa em Enfermagem. Educação de Pós-Graduação em Enfermagem. Brasil.

\begin{abstract}
This is a social-historical study that focuses on the conditions relative to the production of the first dissertations of the Anna Nery Nursing School Master's Degree Program, prepared during the period of 1972 to 1975. Objectives: to describe the initial characteristics of EEAN Master's Degree Program; analyze the profile of the authors of this Program's first dissertations and their advisors; and discuss the insertion of these dissertations in the lines and areas of research in nursing. A documental analysis was accomplished and charts were prepared for the presentation of the results. Results: Nine of the twelve full-time students of the pioneer class submitted their dissertations prior to the final term, which was four years. Although eight of the Master's Degree students were teachers, approximately $90 \%$ of their dissertations are inserted in the Health Care Area, the focus of interest of their teaching practice. The EEAN Master's Degree Program has qualified professionals to teach in higher education nursing courses. The nine first dissertations produced during the program constitute an important contribution to the scientific production of the nursing area.
\end{abstract}

Keywords: History of Nursing. Nursing Research. Education, Nursing, Graduate. Brazil.

\section{Resumen}

Estudio histórico-social que tiene como objeto las condiciones de producción de las primeras disertaciones del Curso de Maestría de la Escuela de Enfermería Anna Nery, elaboradas en el periodo 1972-1975. Objetivos: describir las características iniciales del Curso de Maestría de EEAN; analizar el perfil de las autoras de las primeras disertaciones de ese Curso y de sus orientadoras; y discutir la inserción de esas disertaciones en las áreas y líneas de investigación en enfermería. Se realizó análisis documental y se organizó cuadros para presentación de los resultados. Resultados: Nueve de las doce alumnas de tiempo completo de la clase pionera presentaron sus disertaciones antes del plazo estatutario, que era de cuatro años. Aunque ocho de las alumnas de la maestría fueran profesoras, cerca del $90 \%$ de sus disertaciones se insertan en el Área Asistencial, el foco de interés de su práctica de enseñanza. La maestría de EEAN calificó profesionales para enseñar en los cursos superiores de enfermería. Las nueve primeras disertaciones producidas en el curso constituyeron importante contribución para la producción científica de enfermería.

Palabras clave: Historia de la Enfermería. Investigación en Enfermería. Educación de Postgrado en Enfermería. Brasil.

\footnotetext{
'Enfermeira graduada pela EEAN/UFRJ; Bolsista Pibic/CNPq; Membro efetivo do Núcleo de Pesquisa de História da Enfermagem Brasileira (Nuphebras). Departamento de Enfermagem Fundamental - Escola de Enfermagem Anna Nery - Universidade Federal do Rio de Janeiro- RJ. Brasil. E-mail: carolinarmachado@yahoo.com.br,2Docente do corpo permanente do programa de pós-graduação da EEAN/UFRJ; Membro fundador do Nuphebras; Pesquisador CNPq 1 A. Líder do Grupo de Pesquisa "A prática de enfermagem no Brasil nos anos 60/70 do século XX: assistência, ensino e pesquisa". Departamento de Enfermagem Fundamental - Escola de Enfermagem Anna Nery - Universidade Federal do Rio de Janeiro-RJ. Brasil. E-mail: iedabarreira@openlink.com.br, ${ }^{3}$ Enfermeira; Mestranda da EEAN/UFRJ; Membro efetivo do Núcleo de Pesquisa de História da Enfermagem Brasileira (Nuphebras). Departamento de Enfermagem Fundamental - Escola de Enfermagem Anna Nery - Universidade Federal do Rio de Janeiro - RJ. Brasil. E-mail: analia.martins@gmail.com.
} 


\section{INTRODUÇÃO}

0 presente trabalho tem como objeto de estudo as condições de produção das nove primeiras dissertações produzidas no Curso de Mestrado da Escola de Enfermagem Anna Nery da Universidade Federal do Rio de Janeiro (EEAN/ UFRJ), em um contexto em que a pesquisa de enfermagem era incipiente. 0 recorte temporal tem como marco inicial o ano de 1972, referente ao início de funcionamento do Curso de Mestrado da EEAN e como marco final o ano de 1975, quando são defendidas suas nove primeiras dissertações.

Desde os seus primórdios a EEAN adotara o modelo norte-americano de ensino de enfermagem. Para tanto, 0 cientista Carlos Chagas promovera a vinda de uma missão de enfermeiras americanas à capital do Brasil. Sob a liderança de Ethel Parsons, a missão Parsons permanecera no Rio de Janeiro de 1921 a 1931, com o apoio da Fundação Rockefeller, que desde a formatura da turma pioneira, em 1925, passara a conceder bolsas de estudos a algumas das recém-diplomadas de cada turma, para que se especializassem nos EUA. ${ }^{1}$ Nas décadas seguintes, as diretoras brasileiras da EEAN, preparadas pelas enfermeiras americanas para dar continuidade ao seu trabalho, constituindo assim uma "elite nativa", mantiveram esse modelo de ensino.

As Diretrizes e Bases da Educação Nacional (LDB), regidas pela lei 4.024 / 1961, puseram fim aos adiamentos da exigência do curso secundário completo para as candidatas ao curso de enfermagem, ${ }^{3}$ fazendo avançar o lento processo de inserção da enfermagem na universidade, iniciado em 1937, quando a EEAN ingressara na Universidade do Brasil. ${ }^{4}$

0 contexto político do país em 1964 era o da ditadura militar, propiciada por um golpe civil e militar que depôs o Presidente da República, em um momento de crise econômica e de intensas mobilizações em torno das "reformas de base" defendidas pelo presidente Goulart. ${ }^{5}$

A Reforma Universitária (Lei n ${ }^{0} 5.540 / 1968$ ) adotou o sistema americano de institutos centralizados e organização departamental, fixando o conceito de indissociabilidade entre ensino e pesquisa, do qual decorreu a implantação de cursos de pós-graduação stricto sensu. ${ }^{6}$ Suas intenções declaradas eram o incremento da pesquisa universitária, a qualificação de professores para os cursos de graduação e o preparo de pessoal para empresas públicas e privadas. No entanto, as circunstâncias em que se deu esta reforma impediram o debate e cercearam a liberdade de expressão de professores e estudantes. $^{7}$

As determinações para o provimento das vagas para o magistério superior foram alteradas: os auxiliares de ensino deveriam comprovar, no prazo máximo de quatro anos, a partir da data de sua admissão, serem portadores do grau de mestre; o provimento das vagas de assistentes e adjuntos ficou na dependência do credenciamento de cursos de doutorado ou da abertura de Concursos de Livre Docência. ${ }^{8}$ Esses Concursos foram uma estratégia utilizada pelas universidades públicas para a qualificação de seus professores, tornando-os aptos a lecionar em cursos de pós-graduação stricto sensu. Os diplomas obtidos no concurso tiveram validade legal permanente, o que permitiu não só a qualificação de seus portadores, como também reconhecimento e prestígio. ${ }^{9} 0$ Parecer $n^{0} 77 / 1969$ foi um incentivador para que os docentes defendessem suas teses de Docência Livre e, portanto, se habilitassem à titulação de doutores. $^{6}$

0 Curso de Mestrado da EEAN, o primeiro a ser criado no país (1972), oferecia inicialmente apenas a área de concentração em Enfermagem Fundamental. ${ }^{10} \mathrm{~A}$ primeira turma era constituída por vinte alunas, estando doze em regime de tempo integral.

A influência da enfermagem norte-americana continua a se refletir na implantação do Curso de Mestrado da EEAN, mediada pelas professoras da Escola que haviam realizado cursos de pós-graduação nos EUA ${ }^{3}$ e pela bibliografia de enfermagem adotada no Curso. ${ }^{11}$

0 Curso veio atender à necessidade de qualificação do corpo docente dos cursos de graduação, através da obtenção do título de mestre, ${ }^{12}$ exigido para o magistério superior, sendo a aprovação de uma disser tação requisito indispensável à obtenção do grau de mestre. ${ }^{3}$ Ademais, a enfermagem no Brasil, até à implantação da pós-graduação stricto sensu, ressentia-se da falta de um corpo organizado de conhecimentos, ou pelo menos, do apoio de um quadro referencial devidamente classificado, ordenado, e que pudesse servir de apoio às pesquisas e à elaboração de teses, ${ }^{12}$ uma vez que a produção científica de enfermagem até então abrangia principalmente relatórios sobre "situações-problema". ${ }^{13}$

A criação de mais oito cursos de pós-graduação stricto sensu em enfermagem no Brasil ao longo da década de 1970, distribuídos nas regiões sudeste, nordeste e sul, vem então contribuir decisivamente para o desenvolvimento da pesquisa em enfermagem no país e para a constituição do habitus científico das enfermeiras. ${ }^{4,6}$

A relevância deste estudo prende-se à sua contribuição para a Linha de Pesquisa "A carreira da enfermagem no contexto universitário", pertencente ao Grupo de Pesquisa "A prática de enfermagem no Brasil nos anos 60/70 do século XX: assistência, ensino e pesquisa", coordenado pela Professora Doutora leda de Alencar Barreira.

Foram traçados como objetivos para este estudo:

- descrever as características iniciais do Curso de Mestrado da EEAN;

- analisar o perfil das autoras das nove primeiras dissertações desse Curso e de suas orientadoras;

- discutir a inserção dessas dissertações nas áreas e linhas de pesquisa em enfermagem. 


\section{ABORDAGEM TEÓRICA}

Trata-se de um estudo de natureza histórico-social. Para situar o recorte temporal da pesquisa utilizou-se como referência as fases do saber da enfermagem, segundo a concepção de Almeida e Rocha (1986), ${ }^{14}$ bem como as gerações de pesquisadoras em enfermagem no Brasil, segundo Brandão (1999). ${ }^{15}$

Almeida e Rocha definiram o saber da enfermagem como o instrumental que a enfermagem utiliza para realizar o seu trabalho, pela aproximação e transformação do objeto da enfermagem para alcançar sua finalidade, que é o cuidado do paciente. Este saber é legitimado e reproduzido pelo ensino.

As fases do saber descritas por Almeida e Rocha inserem-se no século XX e são: as técnicas de enfermagem, os princípios científicos e as teorias de enfermagem.

As nove primeiras dissertações do Curso de Mestrado da EEAN foram elaboradas quando predominava a terceira fase do saber da enfermagem. As teorias de enfermagem eram consideradas elementos essenciais à configuração da enfermagem como campo de conhecimento e à busca da autonomia do profissional de enfermagem, por permitir a delimitação de sua atuação, no contexto do trabalho da equipe de saúde. Tais teorias foram desenvolvidas a partir da década de 1960, por enfermeiras norte-americanas, sendo aqui considerados como marco inaugural os conceitos elaborados por Virginia Henderson na década de 1950, fundamentais ao desenvolvimento das teorias de enfermagem. No entanto, 0 surgimento das Teorias de Enfermagem não invalidou os saberes característicos das fases anteriores, que tiveram início nas primeiras décadas do século XX nos Estados Unidos da América do Norte.

No Brasil, nos anos 70, a enfermeira Wanda de Aguiar Horta elaborou a chamada Teoria das Necessidades Humanas Básicas. A autora conceituou a enfermagem como uma ciência aplicada e a arte de assistir ao ser humano no atendimento de suas necessidades básicas, de torná-lo independente desta assistência, quando possível, pelo ensino do autocuidado, de recuperar, manter e promover a saúde em colaboração com outros profissionais. ${ }^{14}$

No que se refere às gerações de enfermeiras, este estudo se insere na época da formação da terceira geração de pesquisadoras, ou seja, as acadêmicas, justamente o grupo de enfermeiras pesquisadoras formadas nos cursos de pósgraduação stricto sensu. No entanto, vale notar que nos anos 70 continuavam em atividade suas antecessoras, as pesquisadoras enfermeiras pioneiras, bem como as autodidatas. ${ }^{15}$

A geração das acadêmicas foi responsável pela criação de pré-condiçõos para o surgimento de grupos de pesquisa com produção científica sistemática e coletiva que viriam a surgir nos anos 90, incrementando o número de publicações, sua inclusão em bibliografias recomendadas nos cursos de enfermagem e citação em publicações de alta qualidade.

\section{METODOLOGIA}

Para o desenvolvimento do estudo foram utilizadas fontes primárias pertencentes ao Centro de Documentação da EEAN (Cedoc/EEAN) e ao acervo da Biblioteca Setorial de Pós-Graduação da EEAN, tais como: memoriais e curricula vitae; Catálogo das disciplinas do Curso de Mestrado em Enfermagem - Anos: 1973/ 74, EEAN/UFRJ; Rol de Pesquisas e Pesquisadores Concluintes do Curso de Mestrado em Enfermagem da EEAN/UFRJ (1972-1981); Programa de Pós-graduação em Enfermagem, elaborado pela Capes/MEC (1977); e as nove dissertaç̃es estudadas.

As fontes secundárias foram artigos, livros e teses referentes ao objeto de estudo, bem como o Banco "Quem é quem na História da Enfermagem Brasileira" (Cedoc/EEAN). As fontes virtuais foram: Plataforma Lattes / CNPq, Biblioteca Virtual de Saúde (BVS/Bireme) e Portal de Periódicos da Capes. Buscas também foram realizadas a partir dos índices remissivos da Revista Brasileira de Enfermagem (Reben).

A coleta de dados concentrou-se no segundo semestre letivo de 2009. 0 instrumento utilizado foi o formulário para coleta de dados sobre o perfil das orientadoras.

Os procedimentos adotados foram a análise documental; a classificação, contextualização e triangulação dos achados com as fontes secundárias, bem como a interpretação dos resultados, à luz dos conceitos de apoio.

\section{RESULTADOS E DISCUSSÃO}

\section{Características Iniciais do Curso de Mestrado da EEAN}

0 Curso de Mestrado, de acordo com a Coordenação do Aperfeiçoamento de Pessoal de Nível Superior (CAPES), visava à qualidade do ensino e da assistência à população e permitir ao estudante o desenvolvimento da capacidade de pensar claramente e de adquirir o conhecimento necessário à sua prática profissional. No entanto, na prática, o que se objetivava era primordialmente o preparo de docentes para o ensino em nível de graduação. ${ }^{10}$

0 Curso veio proporcionar aos profissionais de enfermagem a oportunidade de dar continuidade ao seu processo de qualificação educacional. Este Curso, durante sua implantação, enfrentou limitações de caráter estrutural, tendo porém a seu favor a motivação dos docentes da própria escola e o incentivo da comunidade de enfermagem brasileira. ${ }^{16}$

A EEAN definiu como finalidades do Curso de Mestrado "oferecer oportunidades para o aprofundamento de conhecimentose a ampliação dos níveis de competência e habilidades dos profissionais de enfermagem, não deixando de abranger de forma integrada o ensino, a pesquisa e a assistência de enfermagem". 10:17 Os objetivos do Curso eram os de oferecer ao enfermeiro oportunidades de methor posicionar-se face aos problemas de saúde do indivíduo, da família e da comunidade; de assumir as responsabilidades necessárias à realização de estudos críticos; e ao desenvolvimento de investigações diante das situações de enfermagem. Pretendia ainda o Curso preparar 
os seus estudantes para compreender a relevância do papel do professor no contexto do ensino e da pesquisa de enfermagem, bem como desenvolver as habilidades e criatividade dos estudantes nas estratégias de transmissão de conhecimentos de enfermagem e de abordagem às situações do ensino aplicado. ${ }^{16}$

A coordenadora do Curso de Mestrado à época comenta que o currículo do curso poderia ser considerado assemelhado aos programas de mestrado das universidades americanas, nas duas décadas anteriores, verificando-se as mesmas abordagens, tendências e áreas de concentração. ${ }^{10}$

0 Curso era composto pelas seguintes disciplinas de enfermagem: Enfermagem I - Introdução (90 horas); Enfermagem II - Estudo Crítico (135 horas); Enfermagem III - Avaliação de Enfermagem (90 horas); Ética e Legislação da Enfermagem (90 horas); Orientação e Aconselhamento em Fundamentos de Enfermagem ( 90 horas); e Prática de Ensino de Enfermagem (45 horas). Também eram oferecidas várias disciplinas relacionadas à educação e à prática do ensino, como: filosofia da educação, didática especial, psicologia do desenvolvimento e psicologia educacional. Na distribuição da carga horária curricular, nota-se que $2 / 3$ da carga horária $(810: 1.215$ horas $=67 \%$ ) era destinada às atividades teórico-práticas e à pesquisa, enquanto $1 / 3$ dela $(405: 1.215$ horas $=33 \%)$ era destinada às aulas teóricas. ${ }^{16}$

Quanto ao corpo docente, aquelas professoras que haviam realizado cursos de pós-graduação no exterior, principalmente nos Estados Unidos, foram credenciadas pelo Ministério da Educação como docentes de pós-graduação stricto sensử, como as professoras: Cilei Chaves Rhodus; Elvira de Felice Souza; Haydée Guanais Dourado; Maria Dolores Lins de Andrade; Tereza de Jesus Sena e Vilma de Carvalho. ${ }^{16}$ Também foram credenciadas professoras com cursos de especialização realizados na USP e na UFRJ: Izabel da Cunha Dantas; Maria do Carmo Dantas e Josefa Jorge Moreira.

Professores de outras universidades e de outras áreas, como Educação, Psicologia e Filosofia, que possuíssem qualificação também foram convidados para atuar no Curso de Mestrado. Professores das áreas afins: Dinah Martins de Souza Campos; Hermínio Augusto Faria; Lauro Sollero e Maria Ângela Vinagre de Almeida.

Na opinião de Cilei Chaves Rhodus, coordenadora do Curso de Mestrado da EEAN e também orientadora, o grupo inicial de mestrandas destacou-se por seu espírito de equipe e excelente nível intelectual; além disso, a consciência da responsabilidade de seu pioneirismo tornou-as capazes de vencer as limitações inerentes a um curso apenas em fase de implantação. ${ }^{10}$

0 credenciamento do Curso de Mestrado da EEAN foi estabelecido pelo parecer do Conselho Federal de Educação $n^{0}$ $1.723 / 1973^{12}$. A partir de 1975 , novas áreas de concentração foram desenvolvidas, a saber: Enfermagem Médico-Cirúrgica, Enfermagem Materno-Infantil, Administração de Serviços de Enfermagem e Enfermagem na Saúde da Comunidade.

\section{Perfil das autoras e orientadoras das primeiras dissertações}

0 quadro abaixo apresenta, em ordem de defesa, os títulos das nove primeiras dissertações apresentadas pelas mestrandas da EEAN, suas respectivas instituições de origem e orientadoras.

QUADRO 1: Dissertações de mestrado apresentadas e aprovadas em 1975 na EEAN, segundo a instituição de origem, autores e orientadoras e título da dissertação. Área de Concentração: Enfermagem Fundamental

\begin{tabular}{|c|c|c|}
\hline N de ord end instìuiçāo & $\begin{array}{l}\text { Mestranda (M) } \\
\text { Orientadora (0) }\end{array}$ & Thub da dissertação \\
\hline 1- EEAN & $\begin{array}{l}\text { M- Lygia Paim } \\
\text { 0- Wanda de Aguiar Horta }\end{array}$ & $\begin{array}{l}\text { A prescrição de enfermag em - Unidade valo rativa do } \\
\text { plano de cuidado }\end{array}$ \\
\hline 2- EERP & $\begin{array}{l}\text { M- Ana Maria Palermo da Cunha } \\
0 \text { - Cilei Chaves Rhodus }\end{array}$ & $\begin{array}{l}\text { Avaliação da deg ermação das mãos com } \\
\text { hexaclor ofeno, composto quaternário de amônio e } \\
\text { sabäo comum }\end{array}$ \\
\hline 3- MS/CNCT & $\begin{array}{l}\text { M- leda Barreira e Castro } \\
0 \text { - Vilma de Carvalho }\end{array}$ & $\begin{array}{l}\text { Aspectos críticos do desempenho de funçöes da } \\
\text { enfermeira na assistência ao paciente não } \\
\text { hospitalizado }\end{array}$ \\
\hline 4- EEAN & $\begin{array}{l}\text { M- Solange Maria Ramos } \\
0 \text { - Josefa brge Moreira }\end{array}$ & $\begin{array}{l}\text { A aprend izagem do pacien te diabético - dependência } \\
\text { de en fermagem a nivel de orientaçäo }\end{array}$ \\
\hline 5- EERP & $\begin{array}{l}\text { M- Maura Santesso Takakura } \\
0 \text { - Vilma de Carvalho }\end{array}$ & $\begin{array}{l}\text { Influência da a ssep sia da pele nas in jeções } \\
\text { intramusculares }\end{array}$ \\
\hline 6- EERP & $\begin{array}{l}\text { M- Isab el Amélia Costa Mendes } \\
0 \text { - Izabel da Cunha Dantas }\end{array}$ & $\begin{array}{l}\text { Observação da administração de insulina em } \\
\text { pacientes diabéticos em domicilio }\end{array}$ \\
\hline 7-EERP & $\begin{array}{l}\text { M- Lia Hoelz Ávares } \\
0 \text { - Vilma de Carvalho }\end{array}$ & $\begin{array}{l}\text { A o rienta ção do paciente como função da enfermeira } \\
\text { em uma aplicação em as sistência de enfermag em } \\
\text { cinurgica }\end{array}$ \\
\hline 8- EEAN & $\begin{array}{l}\text { M- Luiza Ap arecida Teike ira Costa } \\
0 \text { - Wanda de Aguiar Horta }\end{array}$ & Situ ações vida-morte. Partic ipação d o enferm eiro \\
\hline 9- EERP & $\begin{array}{l}\text { M- Dulce Maria Vend nuscolo de } \\
\text { Freitas } \\
0 \text { - Cilei Chaves Rhodus }\end{array}$ & $\begin{array}{l}\text { Efeito do banho de imersä0 na incidência de germes } \\
\text { patogênic os no coto umbilical nas primeiras } 24 \text { horas } \\
\text { de vida }\end{array}$ \\
\hline
\end{tabular}

Fontes: Rol de Pesquisas e Pesquisadores Concluintes do Curso de Mestrado em Enfermagem da EEAN/UFRJ (1972-1981); CEDOC/EEAN/ Série Memoriais.

Legenda: EEAN - Escola de Enfermagem Anna Nery/UFRJ; EERP - Escola de Enfermagem de Ribeirão Preto/USP; MS/CNCT Ministério da Saúde/Campanha Nacional Contra a Tuberculose. 
0 Quadro 1 mostra que, das nove mestrandas concluintes do Curso de Mestrado da EEAN em 1975, cinco eram provenientes da Escola de Enfermagem de Ribeirão Preto, enquanto três delas pertenciam à EEAN. Uma mestranda atuava em serviço federal de saúde. A significativa participação de mestrandas da EE de Ribeirão Preto no Curso de Mestrado da EEAN favoreceu o fortalecimento de seu corpo docente. Deste modo, quando o Curso de Mestrado da EERP/USP foi implantado, em 1975, esta Escola já contava com cinco mestras formadas pela EEAN.

Das doze alunas da turma pioneira, matriculadas em 1972 em regime de tempo integral, nove apresentaram suas dissertações antes do prazo previsto de quatro anos. Três dissertações foram aprovadas no dia 20 de maio de 1975, dia do encerramento da Semana Brasileira de Enfermagem. As primeiras enfermeiras a receberem o título de Mestre em Ciência da Enfermagem no Brasil (e na América Latina) foram: Lygia Paim (EEAN/UFRJ), Ana Maria Palermo da Cunha (EERP/USP) e leda Barreira e Castro (Ministério da Saúde). Duas dessas mestras, Lygia Paim e leda Barreira e Castro, fariam o concurso de livre-docência em 1977.

Quatro das cinco orientadoras das nove primeiras dissertações do Curso de Mestrado da EEAN foram ex-alunas e docentes desta Escola, a saber:

1 - Vilma de Carvalho: Piauiense. Diplomada em 1954, foi enfermeira-chefe no Hospital Escola São Francisco de Assis (HESFA), onde também acompanhava o estágio das alunas. Ensinou Enfermagem Médico-Cirúrgica e fez pós-graduação na Universidade de Wayne, em Detroit (Michigan- EUA) de 1961 a 1962, com ênfase em Enfermagem Neurológica. Bacharelou-se em Filosofia na Universidade do Estado do Rio de Janeiro (UERJ). Nessa época já prestara serviços à $A B E n$, como editora da "Revista Brasileira de Enfermagem" (1970-1972) e na assessoria para a revisão do Código de Ética (1973-1974). Participou da implantação do Curso de Mestrado da EEAN e nele lecionou a disciplina Enfermagem Fundamental. ${ }^{17}$ Orientou as mestrandas leda Barreira e Castro, procedente do Ministério da Saúde, Maura Santesso Takakura e Lia Hoelz Álvares, ambas da EERP/USP.

2 - Cilei Chaves Rhodus: Natural do Estado do Rio de Janeiro. Diplomada em 1955, foi enfermeira-chefe no HESFA, onde também acompanhava o estágio das alunas. Ensinou a Arte da Enfermagem nos cursos de auxiliar e técnico de enfermagem. Em 1970 fez pós-graduação naUniversidade do Colorado, em Denver (EUA), sobre Educação da Enfermagem. Participou da implantação do Curso de Mestrado e em 1974 assumiu a coordenação do mesmo. Nele lecionou a disciplina Enfermagem Fundamental ${ }^{17}$. Essa experiência foi objeto de sua tese de livre-docência, defendida em 1977 e intitulada "Pós-graduação em enfermagem, um estudo Curso de mestrado na Escola de Enfermagem Ana Néri da Universidade Federal do Rio de Janeiro". Orientou as mestrandas Ana Maria Palermo da Cunha e Dulce Maria Vendruscolo de Freitas, ambas da EERP/USP.
3 - Izabel da Cunha Dantas: Piauiense. Formada em 1947. Atuou no ensino da Enfermagem de Saúde Pública na EEAN, sendo a primeira chefe deste Departamento. Fez especialização na Faculdade de Higiene da USP. Lecionou no Curso de Mestrado a disciplina Aspectos Sociais e Sanitários no Ensino da Enfermagem ${ }^{16}$. Orientou a mestranda Isabel Amélia Costa Mendes.

4 - Josefa Jorge Moreira: Natural do Rio Grande do Norte. Graduou-se em 1942. Em 1959, recebeu o diploma de Bacharel pela Faculdade de Direito do Distrito Federal. Fez 0 curso de Pedagogia e Didática Aplicada à Enfermagem na EEAN e o de Estudo de Problemas Brasileiros no Fórum de Ciência e Cultura da UFRJ, ambos em 1971. Lecionou as seguintes disciplinas no Curso de Graduação: Estudo de Problemas Brasileiros; Ética; Deontologia, Legislação e Exercício Profissional. Participou da Implantação do Curso de Mestrado, no qual lecionou Estudo de Problemas Brasileiros, disciplina obrigatória por força do decreto-lei n 869/1969, que visava a disseminar a ideologia da segurança nacional, que norteava 0 governo militar. Orientou a mestranda Solange Maria Ramos, pertencente à EEAN.

A única orientadora sem vínculo anterior com a EEAN foi a paraense Wanda de Aguiar Horta, diplomada em 1948 pela EEUSP. Atuou na Secretaria de Saúde do estado do Paraná. Em 1960 tornou-se professora de Fundamentos de Enfermagem da EEUSP, onde desenvolveu estudos sobre os Instrumentos Básicos da Enfermagem e sobre a Metodologia da Assistência de Enfermagem. Em 1968 fez concurso de livre-docência na EEAN/UFRJ com a tese intitulada "A observação sistematizada na identificação dos problemas de enfermagem em seus aspectos físicos", habilitando-se assim para o ensino em cursos de pós-graduação stricto sensu. No Curso de Mestrado da EEAN teve ela a grande responsabilidade de iniciar o ensino do Processo de Enfermagem, no âmbito da disciplina Enfermagem Fundamental. ${ }^{16}$ Orientou as mestrandas Lygia Paim e Luiza Aparecida Teixeira Costa, ambas da EEAN.

\section{As características das nove primeiras dissertações}

A baixa produção científica da enfermagem à época do estudo, segundo Rodrigues (1981), poderia ser explicada por um círculo vicioso, ou seja: não havia produção de pesquisas porque as enfermeiras não eram preparadas para tal prática; por outro lado, a falta de condições favoráveis à realização de pesquisas de enfermagem impedia a preparação de enfermeiras-pesquisadoras. No entanto, o esforço necessário à elaboração dessas dissertações desempenhou um papel relevante na formação inicial de uma comunidade científica de enfermagem, por contribuir tanto para a produção de conhecimento como para o desenvolvimento da capacidade de pesquisar. ${ }^{11}$

A orientação central do Curso de Mestrado da EEAN, voltada para as Teorias de Enfermagem, estava a cargo de 
Wanda de Aguiar Horta, o que explica o fato de que três mestrandas, Lygia Paim, leda Barreira e Castro e Solange Maria Ramos (EEAN) referenciaram a teórica brasileira, utilizando produções suas, como "A metodologia do processo de enfermagem" publicada na Reben $1971^{\mathrm{a}}$ e Reben $1972^{\mathrm{b}}$; e "A observação sistemática na identificação dos problemas de enfermagem em seus aspectos físicos" Reben 1974. Duas mestrandas, Lia Hoelz Álvares (EERP) e Luiza Aparecida Teixeira Costa (EEAN), mesmo que não citassem diretamente as teorias de enfermagem, implicitamente fizeram referência às bases teóricas da metodologia da assistência de enfermagem.

Das nove dissertaç̃oes estudadas, quatro apresentavam temáticas relacionadas aos princípios científicos das técnicas de enfermagem, sendo que duas se referem a procedimentos realizados pela enfermeira no paciente, a saber: "Influência da assepsia da pele nas injeções intramusculares", "Efeito do banho de imersão na incidência de germes patogênicos no coto umbilical nas primeiras 24 horas de vida". Uma se refere a procedimento realizado no paciente por ele mesmo ou por familiar, a saber: "Observação da administração de insulina em pacientes diabéticos em domićlio".

E uma dissertação se relacionava a procedimentos técnicos realizados na enfermeira, por ela mesma: "Avaliação da degermação das mãos com hexaclorofeno, composto quaternário de amônio e sabão comum".

Segundo a classificação das áreas e linhas de pesquisa em enfermagem, produzida no II Seminário Nacional de Pesquisa em Enfermagem (SENPE), de 1982, e publicada nos Anais deste, as nove dissertações em tela abrangem duas das três áreas de pesquisa e no interior destas, quatro das seis linhas de pesquisa, como se vê no Quadro 2.

QUADRO 2: Distribuição das dissertações de mestrado apresentadas e aprovadas em 1975 na EEAN, segundo as áreas e linhas de pesquisa em enfermagem aprovadas no II Seminário Nacional de Pesquisa em Enfermagem (Brasília, 1982)

\begin{tabular}{|c|c|c|}
\hline Área de Pesquisa & Linha de Pesquisa & Título da Dissertação \\
\hline \multirow[t]{2}{*}{ I. Profissional } & 1. A enfermagem como prática social & $\begin{array}{l}\text { Aspectos críticos do desempenho de funções da enfermeira na } \\
\text { assistência ao paciente não hospitalizado }\end{array}$ \\
\hline & $\begin{array}{l}\text { 2. Formação e utilização dos recursos } \\
\text { humanos em enfermagem }\end{array}$ & - \\
\hline \multirow[t]{9}{*}{ II. Assistencial } & \multirow[t]{4}{*}{$\begin{array}{l}\text { 1.Fundamentação da Assistência, Tecnologia } \\
\text { e Instrumentação }\end{array}$} & $\begin{array}{l}\text { A prescrição de enfermagem - Unidade valorativa do plano de } \\
\text { cuidado }\end{array}$ \\
\hline & & $\begin{array}{l}\text { Avaliação da degermação das mãos com hexaclorofeno, } \\
\text { composto quaternário de amônio e sabão comum }\end{array}$ \\
\hline & & Influência da assepsia da pele nas injeções intramusculares \\
\hline & & $\begin{array}{l}\text { Efeito do banho de imersão na incidência de germes } \\
\text { pato gênicos no coto umbilical nas primeiras } 24 \text { horas de vida }\end{array}$ \\
\hline & \multirow[t]{3}{*}{ 2.Autocuidado à saúde } & $\begin{array}{l}\text { A aprendizagem do paciente diabético - dependência de } \\
\text { enfermagem a nível de orientação }\end{array}$ \\
\hline & & $\begin{array}{l}\text { Observação da administração de insulina em pacientes } \\
\text { diabéticos em domićlio }\end{array}$ \\
\hline & & $\begin{array}{l}\text { A orientação do paciente como função da enfermeira numa } \\
\text { aplicação em assistência de enfermagem cirúrgica }\end{array}$ \\
\hline & 3.Riscos da assistência de enfermagem & - \\
\hline & 4.Determinantes do processo saúde-doença & Situações vida-morte. Participação do enfermeiro \\
\hline \multirow{5}{*}{$\begin{array}{l}\text { III. Estrutura, } \\
\text { Organização e } \\
\text { Funcionamento das } \\
\text { Instituições de Saúde }\end{array}$} & 1.Modelos de assistência de enfermagem & - \\
\hline & $\begin{array}{l}\text { 2.Oferta, acessibilidade e utilização de } \\
\text { serviços de saúde }\end{array}$ & - \\
\hline & 3.Sistema de informação em enfermagem & - \\
\hline & $\begin{array}{l}\text { 4.Incorporação de conhecimentos à prática } \\
\text { profissional }\end{array}$ & - \\
\hline & 5.Dinâmica das organizações & - \\
\hline
\end{tabular}

A Área I - Profissional foi representada apenas pelo estudo da mestranda vinculada ao Ministério da Saúde, na função de supervisora da Campanha Nacional Controle a Tuberculose. Seu estudo se insere na linha 1: A enfermagem como prática social e abordou uma temática mais próxima à sua experiência profissional, qual seja, "Aspectos críticos do desempenho de funções da enfermeira na assistência ao paciente não hospitalizado".

Na Área II - Assistencial, de maior predomínio, com $89 \%$ das dissertações, enquadraram-se oito destas, pertencentes a mestrandas que atuavam na docência. Suas pesquisas distribuem-se por três das quatro linhas de pesquisa 
dessa área. Assim, quatro inserem-se na linha 1: Fundamentação da Assistência, Tecnologia e Instrumentação; três inserem-se na linha 2: Autocuidado à saúde; e uma se insere na linha 4: Determinantes do processo saúde-doença. Nenhuma dissertação se inseriu na linha 3: Riscos da assistência de enfermagem, que viria a se desenvolver em anos posteriores.

0 maior interesse das mestrandas em abordar questões ligadas à assistência pode estar associado à sua necessidade de explicar os princípios científicos dos procedimentos técnicos abordados em sua prática de ensino.

A ausência de trabalhos na Área III - Estrutura, Organização e Funcionamento das Instituições de Saúde talvez possa ser atribuída a uma possível falta de experiência relacionada a essa área, por parte das mestrandas e de suas orientadoras, cujo interesse estaria mais voltado para os aspectos internos da prática da enfermagem.

Os achados desse trabalho, relacionados à distribuição das nove dissertações entre as três áreas de pesquisa, corroboram o resultado encontrado por Baptista ${ }^{18}$ sobre as dissertações de mestrado na EEAN no período 1975-1981, onde se observa o predomínio da Área II - Assistencial em um universo de 99 dissertações. A autora atribui a predominância dos estudos dessa Área a uma maior preocupação com a assistência em enfermagem e que às mestrandas interessava questionar elementos substantivos da prática profissional e reconsiderar o papel e as funções do enfermeiro.

\section{CONCLUSÕES}

A obtenção do grau de mestre mediante a apresentação de uma dissertação significou a titulação e qualificação de enfermeiros para lecionar nos cursos superiores de enfermagem, minimizando a escassez de docentes para o acesso à carreira do magistério superior, segundo exigência da reforma universitária de 1968.

A elaboração das nove primeiras dissertações de mestrado em enfermagem representou um incremento da produção científica de enfermagem, constituindo-se em uma resposta positiva ao contexto de criação dos cursos de mestrados no país, contribuindo para que as enfermeiras brasileiras enveredassem, mais efetivamente, pelos caminhos da pesquisa. Representaram ainda o fortalecimento do corpo docente da EEAN/UFRJ e da EERP/USP ao serem incorporadas a estes as primeiras mestras do país.

Quatro das cinco orientadoras dessas dissertações eram docentes da EEAN, onde se haviam graduado, com exceção de Wanda Horta. Três orientadoras estiveram diretamente ligadas ao processo de implantação do Curso de Mestrado da EEAN.

Apesar das nove dissertações emergirem durante a terceira fase do saber de enfermagem, caracterizada pelas Teorias de Enfermagem, quatro dissertações evidenciaram uma abordagem relacionada à fase anterior, que se refere aos Princípios Científicos, elemento central de sua prática de ensino.

Da mesma forma, as dissertações analisadas inseremse principalmente na Área Assistencial, demonstrando uma preferência das mestrandas, docentes em suas Escolas de Enfermagem, em estudar questões relacionadas à sua prática de ensino.

\section{REFERÊNCIAS}

1.Sauthier J. As enfermeiras norte-americanas e o ensino da enfermagem na capital do Brasil: 1921-1931. Rio de Janeiro: EEAN/UFRJ; 1999.

2.Santos TCF, Barreira IA. A mulher e a enfermeira na nova ordem social do Estado Novo. Texto\&Contexto Enferm. 2008 jul/set; 17(3): 587-93.

3.BaptistaSS, Barreira IA. Docentes e estudantes no processo de mudança do ensino de enfermagem no Brasil. Texto\&Contexto Enferm. 1999 jan/ $\mathrm{abr} ; 8(1): 67-79$.

4.Almeida MCP, Barreira IA. Os estudos de pós-graduação em enfermagem na América Latina: sua inserção na comunidade cientifica. In: Sena RR, organizadora. Educación de enfermería en América Latina. Bogotá: Universidad Nacional de Colombia/Rede de Enfermería de América Latina; 2000. p.129-45.

5. Habert N. A década de 70: apogeu e crise da ditadura militar brasileira. São Paulo: Ática; 1992.

6.Santos TCF, Gomes MLB. Nexos entre pós-graduação e pesquisa em enfermagem no Brasil. Rev Bras Enferm. [periódico on-line] 2007 jan/ fev; [citado 2009 ago 15]; 60(1):[aprox. 4 telas]. Disponível em: http:/ /www.scielo.br.

7.Baptista SS, Barreira IA. Repercussões da reforma universitária de 1968 nas escolas de enfermagem brasileiras. Acta Paul Enferm. 1999 set/dez; 12(3): 46-50.

8.Andrade MDL, Rhodus CC, Souza EF, Paim L, Carvalho V. 0 curso de graduação da Escola de Enfermagem Anna Nery da UFRJ - Currículo Pleno face ao parecer 163/72. Rev Bras Enferm. 1973 jul/set; 16(4/5): 253-272.

9.Martins ALT, Barreira IA, Baptista SS. Concursos de livre-docência na Escola de Enfermagem Anna Nery: estratégia de qualificação de professores. Enferm Atual. 2010 mar/abr; v. 10(56): 10-12.

10.Rhodus CC. Pós-graduação em enfermagem, um estudo. Curso de mestrado na Escola de Enfermagem Ana Néri da Universidade Federal do Rio de Janeiro [tese de livre docência]. Rio de Janeiro: Escola de Enfermagem Anna Nery, Universidade Federal do Rio de Janeiro; 1977.

11.Rodrigues APS. Possibilidades e limitações da pesquisa em enfermagem no Brasil; estudo comparativo entre mestras e mestrandas e suas dissertações de mestrado. [dissertação]. Rio de Janeiro: Escola de Enfermagem Anna Nery, Universidade Federal do Rio de Janeiro; 1981. 
12.Tyrrell MAR, Batista SS, Barreira IA, Carvalho V. Programa de pósgraduação em enfermagem da Escola de Enfermagem Anna Nery Universidade Federal do Rio de Janeiro: inovações curriculares no curso de mestrado. Rev Bras Enferm. 1993 jan/mar; 46(1):72-79.

13.Miranda CML, Carvalho V, Souza EF. Integração docente assistencial: uma questão "não resolvida". Esc Anna Nery 1997 dez; 1(2): 72-83.

14.Almeida MCP, Rocha JSY. 0 saber de enfermagem e sua dimensão prática. São Paulo: Cortez; 1986.

15.Brandão EM. A formação do campo científico de enfermagem no Brasil: sociologia da geração de pesquisadoras pioneiras, 1935-1958. [tese]. Rio de Janeiro: Escola de Enfermagem Anna Nery, Universidade Federal do Rio de Janeiro; 1999.

16. Rhodus CC, Andrade MDL, Sena TJ. Evolução curricular do Curso de Mestrado da EEAN/UFRJ. Esc Anna Nery 1988; 1(1): 17-27. (Anais do Seminário de Avaliação do Curso de Mestrado EEAN/UFRJ).

17.Coelho CP. EEAN: suas histórias, nossas memórias. Rio de Janeiro: Cultura Médica; 1997.

18.Baptista SS. Tendências da produção científica do curso de mestrado em enfermagem da Escola de Enfermagem Anna Neri/UFRJ: estudo das teses aprovadas no período de 1975-1981. [dissertação]. Rio de Janeiro: Escola de Enfermagem Anna Nery, Universidade Federal do Rio de Janeiro; 1983.

\section{NOTAS}

aHorta WA. A metodologia do processo de enfermagem. Rev bras enferm. 1971 out/dez; 24(6): 81-95.

${ }^{b}$ Horta WA. A metodologia do processo de enfermagem. Rev bras enferm. 1972 jan/abr; XXV (1 e 2):14-26.

'Horta WA. A observação sistemática na identificação dos problemas de enfermagem em seus aspectos físicos. Rev bras enferm. 1974 abr/jun; 2: 214-219. 